\title{
GS305+04-26: revisiting the interstellar medium around the Centaurus OB1 stellar association
}

\author{
M. A. Corti ${ }^{1,2, \star}$, E. M. Arnal ${ }^{1,2, \star}$, and R. B. Orellana ${ }^{2,3, \star}$ \\ 1 Instituto Argentino de Radioastronomía, CCT-La Plata, CONICET, C.C. No. 5, 1894 Villa Elisa, Argentina \\ e-mail: mariela@fcaglp.unlp.edu.ar \\ 2 Facultad de Ciencias Astronómicas y Geofísicas, Universidad Nacional de La Plata, Paseo del Bosque s/n, 1900 La Plata, Argentina \\ 3 Instituto de Astrofísica de La Plata, CCT-La Plata, CONICET, Argentina
}

Received 14 November 2011 / Accepted 31 July 2012

\begin{abstract}
Context. Massive stars deeply modify their surrounding interstellar medium (ISM) via their high throughput of ionizing photons and their strong stellar winds. In this way, they may create large expanding structures of neutral gas.

Aims. We study a new large neutral hydrogen (HI) shell, labelled GS305+04-26, and its relationship with the OB association Cen OB1. Methods. To carry out this study, we have used a multi-wavelength approach. We analyse both Hi line data retrieved from the LeidenArgentina-Bonn (LAB) survey and new spectroscopic optical observations obtained at CASLEO, as well as make use of proper motion databases available from CDS.

Results. The analysis of the Hi data reveals a large expanding structure GS305+04-26 centred at $(l, b)=\left(305^{\circ},+4^{\circ}\right)$ in the velocity range from -33 to $-17 \mathrm{~km} \mathrm{~s}^{-1}$. Based on its central velocity of $-26 \mathrm{~km} \mathrm{~s}^{-1}$ and using standard galactic rotation models, a distance of $2.5 \pm 0.9 \mathrm{kpc}$ is inferred. This structure, elliptical in shape, has a major and minor axis of 440 and $270 \mathrm{pc}$, respectively. Its expansion velocity, total gaseous mass, and kinetic energy are $\sim 8 \mathrm{~km} \mathrm{~s}^{-1},(2.4 \pm 0.5) \times 10^{5} M_{\odot}$, and $(1.6 \pm 0.4) \times 10^{50} \mathrm{erg}$, respectively. Several stars of the OB-association Cen OB1 are seen projected onto, and within, the boundaries of GS305+04-26. Based on an analysis of proper motions, new members of Cen OB1 are identified. The mechanical energy injected by these stars could have been the origin of this Hi structure.
\end{abstract}

Key words. Galaxy: kinematics and dynamics - Galaxy: structure - open clusters and associations: individual: Cen OB1 ISM: bubbles - ISM: structure - radio lines: ISM

\section{Introduction}

Hi emission at $\lambda \sim 21-\mathrm{cm}$ shows that the ISM of spiral galaxies have a complex morphology, because a plethora of different features (arcs, bubbles, chimneys, filaments, holes, loops, shells, supershells, worms, etc.) are observed superimposed on their large-scale structure. Among them, large Hi shells and their major siblings, the so-called Hi supershells, are some of the most spectacular phenomena. Though the origin of the large shells can well be explained by the action of either stellar winds or supernova explosions, or most likely, by their combined effects, the origin of the Hi supershells is not well understood yet. The supershells were discovered by Heiles (1979, 1984). Large shells of Hi and Hi supershells are usually identified, in a given velocity range, as a minimum in the Hi emissivity distribution surrounded by regions of higher emissivity. In order to power the Hi supershells, an unrealistically large number of massive stars, ranging from hundreds to thousands, would be required. For these extreme cases, alternative processes like gamma-ray bursts (Perna \& Raymond 2000) or the infall of high-velocity clouds (Tenorio-Tagle 1981) have been invoked. Nevertheless, a particular OB-association has been identified as the likely powering source for a handful of the catalogued Hi supershells. McClure-Griffiths et al. (2001) suggest that the Hi supershell GSH305+01-24 was "formed from stellar winds in the Centaurus OB1 association". It is worth mentioning

\footnotetext{
* Member of Carrera del Investigador Científico, CONICET-CCT La Plata, Argentina.
}

that to reach this conclusion they considerer the Wolf-Rayet star WR 48 ( $\theta$ Mus) to be a member of the association. As an argument in favour of such interpretation, the authors say that "the morphology of the shell seems to trace the stellar distribution, suggesting an association".

As part of a long-term program aimed at improving the optical data (e.g. radial velocity, binarity, spectral types, membership) of stars considered to be members of OB-associations located in the Southern Hemisphere, new spectroscopic observations were carried out toward most of the early-type stars listed by Humphreys (1978) as members of Cen OB1. Furthermore, by using the Tycho-2 catalogue and applying a new method of proper motion analysis recently developed by Orellana et al. (2010), a substantial revision of the stars thought to be members of Cen OB1 was made.

In light of the above results, it was found that the census of early-type stars, those that matter from a wind-injection energy viewpoint, has been altered to such an extent that it is worthwile to review the suggested association between Cen OB1 and GSH305+01-24 claimed by McClure-Griffiths et al. (2001).

\section{Observational data}

This research was carried out using both new observational data and databases publicly available, namely:

a) Spectroscopic observations of 16 stars listed by Humphreys (1978) as members of Cen OB1 were acquired at Complejo 
Table 1. New spectral types and radial velocity measurements of Cen OB1 members (according to Humphreys 1978).

\begin{tabular}{lccccccc}
\hline \hline ID & $\begin{array}{c}l \\
(\circ)\end{array}$ & $\begin{array}{c}b \\
(\mathrm{o})\end{array}$ & SpT & $\begin{array}{c}V \\
(\mathrm{mag} .)\end{array}$ & $\begin{array}{c}V_{\text {LSR }} \\
\left(\mathrm{km} \mathrm{s}^{-1}\right)\end{array}$ & $\begin{array}{c}d \\
(\mathrm{kpc})\end{array}$ & Commentaries \\
\hline HD 110639 & 302.1 & +1.5 & B1 II-III & 8.5 & $-52 \pm 03$ & $1.8 \pm 0.5$ & \\
HD 111613 & 303.0 & +2.5 & B9-A0 Iab & 5.7 & $-27 \pm 03$ & $1.5 \pm 0.4$ & \\
HD 111904 & 303.2 & +2.5 & B9 Ia & 5.7 & $-25 \pm 03$ & $2.6 \pm 0.6$ & NGC 4755 \\
HD 111934 & 303.2 & +2.5 & B2 Ib & 7.0 & $-31 \pm 02$ & $2.0 \pm 0.5$ & NGC 4755 \\
HD 111973 & 303.2 & +2.5 & B5 Ia & 5.9 & $-18 \pm 04$ & $2.7 \pm 0.6$ & NGC 4755 \\
HD 111990 & 303.2 & +2.5 & B3 Ib & 6.8 & $-7 \pm 03$ & $1.8 \pm 0.5$ & NGC 4755 \\
HD 112364 & 303.6 & +3.1 & B0.5 Ib-a & 7.4 & $-68 \pm 12$ & $2.8 \pm 0.6$ & SB2 \\
HD 112842 & 304.1 & +2.5 & B3 Ib & 7.0 & $-33 \pm 03$ & $2.1 \pm 0.5$ & \\
HD 113012 & 304.3 & +2.7 & B0 Ia & 8.1 & $-22 \pm 05$ & $6.9 \pm 1.6$ & \\
HD 113422 & 304.5 & +1.1 & B1 Ia & 8.2 & $-57 \pm 04$ & $3.2 \pm 0.7$ & \\
HD 113432 & 304.4 & -0.7 & B1 Ib & 8.9 & $-21 \pm 11$ & $2.7 \pm 0.6$ & \\
HD 113708 & 304.6 & -2.4 & B0.2 III & 8.1 & $-16 \pm 07$ & $3.1 \pm 0.8$ & \\
HD 114213 & 305.2 & +1.3 & B1 Ia-b & 8.9 & $-2 \pm 06$ & $2.2 \pm 0.5$ & \\
HD 114886 & 305.6 & -0.9 & O9 II-III & 6.9 & $-25 \pm 08$ & $2.6 \pm 0.6$ & SB2 \\
HD 115363 & 305.9 & -1.0 & B1 Ia & 7.8 & $-66 \pm 08$ & $3.3 \pm 0.9$ & \\
HD 116119 & 306.7 & +0.6 & B8 Ia & 7.9 & $-34 \pm 03$ & $4.3 \pm 1.1$ & \\
\hline
\end{tabular}

Astronómico El Leoncito (CASLEO) ${ }^{1}$ during April 2009. The spectra were obtained using a REOSC Cassegrain echelle spectrograph attached to the $2.15 \mathrm{~m}$ "Jorge Sahade" telescope. The detector was a TEK CCD $(1024 \times 1024$ pixels) with a pixel size of $24 \mu \mathrm{m}$. A grating of $400 \mathrm{l} / \mathrm{mm}$ was used as cross disperser, and the slit width was set either to $250 \mu \mathrm{m}$ or $300 \mu \mathrm{m}$. The reciprocal dispersion was $6.6 \AA / \mathrm{mm}$. These spectra covered a wavelength range from $3800 \AA$ to $6500 \AA$, and the signal-to-noise ratio was $20 \leq S / N \leq 50$.

Th-Ar comparison lamp images were obtained at the same telescope position as the stellar images immediately after or before the stellar exposures. Bias frames were also obtained every night, as well as spectra of the stars HR 2806 and HR 7773 as radial velocity standards. In addition, the spectra of several spectral type standards were obtained along the observing run. All spectra were processed and analysed using the IRAF ${ }^{2}$ package.

b) HI line data retrieved from the Leiden-Argentina-Bonn (LAB) survey (Kalberla et al. 2005).

c) WR 48 proper motion measurements retrieved from the Centre de Données Astronomiques de Strasbourg $\left(\mathrm{CDS}^{3}\right)$.

\section{Results}

\subsection{Optical spectroscopic}

Most of the Cen OB1 stars listed by Humphreys (1978) with spectral types earlier than A2 were observed using the CASLEO telescope. They are listed in Table 1. Using these data and the spectral type catalogue of Walborn \& Fitzpatrick (1990), a new spectral classification was derived for each star (see fourth column in Table 1). The star identification is given in the first column, whilst its galactic coordinates (longitude and latitude) are given in the second and third columns, respectively. Using this new spectral type, the intrinsic $(B-V)_{0}$ colour index and the absolute visual magnitude $M_{\mathrm{v}}$ matching the spectral type were obtained from Schmidt-Kaler (Th. 1982). With these parameters

\footnotetext{
${ }^{1}$ Operated under agreement between CONICET, SeCyT, and the Universities of La Plata, Córdoba and San Juan, Argentina.

2 IRAF is distributed by NOAO and operated by AURA, Inc. under an agreement with NSF.

${ }^{3}$ http://cdsweb.u-strasbg.fr/
}

and adopting a visual extinction $A_{\mathrm{v}}=3.26 E(B-V)$, where $E(B-V)$ is the stellar colour excess, the stellar distance (seventh column in Table 1) of each star is derived. The photometric magnitudes $V$ and $B$ were corrected from the Tycho- $2^{4}$ system to the Johnson system. The quoted error in the distances stems from an assumed $M_{\mathrm{v}}$ uncertainty of $0.5 \mathrm{mag}$ (Walborn 1972).

Using the IRAF standard reduction package, the stellar heliocentric radial velocity was derived from a Gaussian fit to the He I and He II lines present in the stellar spectrum. Later on, this heliocentric radial velocity was corrected to the local standard of rest (LSR) (see sixth column, Table 2). Since all program stars but two (HD 113432 and HD 114213) were observed twice, stars with highly discordant individual radial velocity estimates were identified as possible multiple (SB2 or triple) systems. Based on the radial velocity obtained for the stars assumed to be single, the mean radial velocity of Cen OB1 is $-24 \pm 14 \mathrm{~km} \mathrm{~s}^{-1}$. Throughout this paper, all radial velocities are referred to the LSR.

\subsection{Proper motion analysis}

\subsubsection{WR 48}

Large-scale catalogues (Hipparcos 1997; Tycho2 2000; PPMX 2008; ASCC2.5 2009) quote proper motions for the Wolf-Rayet

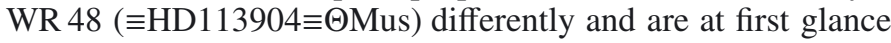
even contradictory. All proper motions in this paper will be expressed in units of milliarcseconds per year (mas/yr). From a thorough analysis of the proper motions provided for this star by the different catalogues, those given by the ASCC 2.5 catalogue $\left(\mu_{\alpha} \cos \delta, \mu_{\delta}\right)=(-4.42 \pm 0.79,2.53 \pm 0.95)($ Kharchenko 2001) are found to be the most reliable and will be adopted in this paper. This conclusion is based on the following considerations:

a) It is a well-known fact that due to the short time interval covered by the HipParcos mission (less than four years), the proper motions provided by this catalogue are often not as good as their formal standard errors seem to indicate. This situation is even worse for double or multiple stars (Urban et al. 2000; Vondrak 2004), because in these cases the quoted proper motions may reflect the instantaneous proper motion

\footnotetext{
${ }^{4}$ http://www.rssd.esa.int/index.php? page=0verview\&project $=$ HIPPARCOS
} 
M. A. Corti et al.: GS305+04-26: revisiting the ISM around the Cen OB1 stellar association

Table 2. Information about the possible members of Centaurus OB1.

\begin{tabular}{|c|c|c|c|c|c|c|c|c|c|}
\hline ID & $\begin{array}{l}\lg \\
(\circ)\end{array}$ & $\begin{array}{l}\text { bg } \\
\text { (०) }\end{array}$ & SpT & $\begin{array}{c}V \\
(\mathrm{mag})\end{array}$ & $\begin{array}{c}\mathrm{RV}(L S R) \\
\left(\mathrm{km} \mathrm{s}^{-1}\right)\end{array}$ & $\begin{array}{c}d \\
(\mathrm{kpc})\end{array}$ & $\begin{array}{c}M \\
\left(M_{\odot}\right)\end{array}$ & $\begin{array}{c}{ }^{t} \\
\left(10^{6} \mathrm{yrs}\right)\end{array}$ & Comments \\
\hline HD 108516 & 300.3 & -0.2 & B2 III & 9.5 & & $2.8 \pm 0.6$ & 12.0 & 19.0 & \\
\hline HD 109150 & 300.6 & 3.3 & B2 II-III & 8.6 & & $2.9 \pm 0.7$ & 14.5 & 14.0 & \\
\hline HD 109253 & 301.0 & -1.6 & B8 II-III c & 9.6 & & $2.3 \pm 0.5$ & - & - & \\
\hline HD 109505 & 301.1 & 1.2 & B2 II c & 8.0 & & $2.0 \pm 0.5$ & 17.0 & 11.0 & \\
\hline HD 109937 & 301.6 & -0.5 & B2-3 III & 9.4 & & $2.0 \pm 0.5$ & 10.5 & 24.0 & \\
\hline HD 109978 & 301.6 & 0.0 & O9IV & 8.9 & $-13 \pm 5^{(3)}$ & $2.6 \pm 0.6$ & 22.0 & 8.1 & \\
\hline HD 110449 & 301.9 & 1.9 & B2 III c & 9.3 & & $2.5 \pm 0.6$ & 12.0 & 19.0 & \\
\hline HD 110597 & 302.0 & 3.5 & B8 II & 9.5 & & $2.3 \pm 0.5$ & 11.4 & 21.0 & \\
\hline CD-64 654 & 302.3 & -2.7 & B1 V & 9.7 & & $2.2 \pm 0.5$ & 13.4 & 16.0 & \\
\hline HD 110912 & 302.3 & 2.1 & B8 II-III & 9.8 & & $2.2 \pm 0.5$ & - & - & \\
\hline HD 110984 & 302.4 & 1.7 & B1 II-III & 9.0 & & $2.0 \pm 0.5$ & 20.0 & 9.2 & \\
\hline HD 111121 & 302.5 & 3.8 & B8-9 II-III & 9.6 & & $2.7 \pm 0.6$ & - & - & \\
\hline HD 111579 & 302.8 & 1.6 & B2 Ib-II & 9.1 & & $2.0 \pm 0.5$ & 19.5 & 9.5 & \\
\hline CP-59 4552 & 303.2 & 2.5 & B1 III & 8.2 & $-21^{(3)}$ & $2.0 \pm 0.5$ & 15.4 & 13.0 & NGC $4755^{(2)}$ \\
\hline HD 111904 & 303.2 & 2.5 & B9 $\mathrm{Ia}^{(1)}$ & 5.8 & $-25 \pm 3^{(1)}$ & $2.5 \pm 0.6$ & 16.6 & 12.0 & NGC $4755^{(2)}$ \\
\hline HD 111934 & 303.2 & 2.5 & $\mathrm{~B} 2 \mathrm{Ib}^{(1)}$ & 6.9 & $-31 \pm 2^{(1)}$ & $2.0 \pm 0.5$ & 22.0 & 8.1 & NGC $4755^{(2)}$ \\
\hline HD 111973 & 303.2 & 2.5 & B5 $\mathrm{Ia}^{(1)}$ & 5.9 & $-18 \pm 4^{(1)}$ & $2.6 \pm 0.6$ & 20.0 & 9.2 & NGC $4755^{(2)}$ \\
\hline HD 112026 & 303.3 & 2.0 & B0.5 IV & 8.7 & & $2.7 \pm 0.6$ & 16.3 & 12.0 & \\
\hline HD 112147 & 303.4 & 3.8 & B0 IV pe & 9.1 & & $2.9 \pm 0.7$ & 18.7 & 10.0 & \\
\hline HD 112181 & 303.4 & 2.2 & B2-3 II-III & 8.8 & & $2.0 \pm 0.5$ & 13.4 & 16.0 & \\
\hline HD 112364 & 303.6 & 3.1 & B0.5 Ib-a ${ }^{(1)}$ & 7.4 & $-68 \pm 12^{(1)}$ & $2.8 \pm 0.6$ & 25.0 & 7.0 & SB2 \\
\hline HD 112366 & 303.6 & -0.6 & B6 Iab-b & 7.6 & $-07 \pm 2^{(3)}$ & $1.9 \pm 0.4$ & 19.0 & 9.8 & \\
\hline HD 112382 & 303.6 & 3.8 & B4 II & 9.0 & & $2.5 \pm 0.6$ & 15.0 & 14.0 & \\
\hline HD 112471 & 303.7 & 2.0 & B1 II-III & 8.8 & & $2.4 \pm 0.5$ & 20.0 & 9.2 & \\
\hline HD 112485 & 303.7 & 2.0 & B2 III & 9.5 & $-15^{(3)}$ & $2.9 \pm 0.7$ & 12.0 & 19.0 & \\
\hline HD 112497 & 303.7 & 1.8 & B1-2 II-III & 8.4 & & $2.1 \pm 0.5$ & 15.9 & 12.0 & \\
\hline HD 112637 & 303.8 & -0.4 & B2 III c & 9.6 & & $2.1 \pm 0.5$ & 12.0 & 19.0 & \\
\hline HD 112661 & 303.8 & 0.6 & B0.5 III-IV & 9.3 & & $1.9 \pm 0.4$ & 17.0 & 11.3 .0 & \\
\hline HD 112784 & 304.0 & 2.3 & O9.5 III & 8.3 & $-34 \pm 5^{(3)}$ & $3.2 \pm 0.7$ & 21.7 & 8.3 & \\
\hline HD 112842 & 304.1 & 2.5 & B3 $\mathrm{Ib}^{(1)}$ & 7.1 & $-33 \pm 3^{(1)}$ & $2.1 \pm 0.5$ & 21.1 & 8.6 & \\
\hline HD 112852 & 304.1 & 3.3 & B8-9 II & 9.9 & & $2.6 \pm 0.6$ & 11.0 & 22.0 & \\
\hline HD 113421 & 304.6 & 3 & B0 Vn & 9.4 & $-33^{(3)}$ & $2.5 \pm 0.6$ & 17.5 & 11.0 & \\
\hline HD 113422 & 304.5 & 1.1 & $\mathrm{~B} 1 \mathrm{Ia}^{(1)}$ & 8.3 & $-57 \pm 4^{(1)}$ & $3.2 \pm 0.7$ & 23.0 & 7.7 & \\
\hline HD 113432 & 304.4 & -0.7 & $\mathrm{~B} 1 \mathrm{Ib}^{(1)}$ & 8.9 & $-21 \pm 11^{(1)}$ & $2.7 \pm 0.6$ & 23.0 & 7.7 & \\
\hline HD 113606 & 304.5 & -1.4 & $\mathrm{O} 7$ & 8.7 & & $2.2 \pm 0.5$ & 31.4 & 5.6 & \\
\hline HD 113742 & 304.7 & 0.9 & B1-2 III & 9.2 & & $2.3 \pm 0.5$ & 13.7 & 16.0 & \\
\hline HD 113754 & 304.7 & -0.2 & O6-7 & 9.5 & $-25 \pm 2^{(3)}$ & $2.6 \pm 0.6$ & 34.2 & 5.2 & \\
\hline СР-61 3452 & 304.8 & 1.0 & O9.5 II c & 9.3 & & $2.6 \pm 0.6$ & 22.0 & 8.1 & \\
\hline HD 114011 & 305.0 & 1.6 & B4 Ib-II & 9.3 & $-34 \pm 5^{(3)}$ & $2.9 \pm 0.7$ & 17.7 & 11.0 & \\
\hline HD 114213 & 305.2 & 1.3 & B1 Ia-b ${ }^{(1)}$ & 8.9 & $-02 \pm 6^{(1)}$ & $2.2 \pm 0.5$ & 23.0 & 7.7 & \\
\hline HD 114394 & 305.4 & 3.2 & B1 III & 8.2 & & $2.0 \pm 0.5$ & 16.3 & 12.0 & \\
\hline HD 114478 & 305.3 & 0.0 & B1 Ib-II & 8.7 & $-1^{(3)}$ & $2.6 \pm 0.6$ & 21.1 & 8.6 & \\
\hline HD 114669 & 305.4 & 0.2 & B5 II & 9.1 & & $2.6 \pm 0.6$ & 14.5 & 14.0 & \\
\hline HD 114737 & 305.4 & 0.1 & O9 III & 8.0 & & $2.7 \pm 0.6$ & 23.4 & 7.6 & \\
\hline CP-60 4528 & 305.5 & 1.7 & B1 III & 8.8 & $-25^{(3)}$ & $2.2 \pm 0.5$ & 15.4 & 13.0 & \\
\hline CP-60 4551 & 305.8 & 1.8 & B1 III (N)e & 9.8 & & $2.3 \pm 0.5$ & 15.4 & 13.0 & \\
\hline HD 115223 & 306.4 & 3.7 & A0 Ib-II & 8.7 & & $2.8 \pm 0.6$ & 13.0 & 17.0 & \\
\hline HD 115455 & 306.1 & 0 & O7.5 III & 8.0 & $-44 \pm 14^{(3)}$ & $3.0 \pm 0.7$ & 29.0 & 6.0 & \\
\hline HD 115497 & 306.4 & 3.7 & B8-9 II & 9.7 & & $2.8 \pm 0.6$ & 11.0 & 22.0 & \\
\hline CP-61 3576 & 306.1 & 0.2 & B $0.5 \mathrm{~V}$ & 9.5 & $-40 \pm 69^{(3)}$ & $2.1 \pm 0.5$ & 14.9 & 13.7 & \\
\hline HD 115666 & 306.4 & 1.8 & B7 IIc & 9.4 & & $2.8 \pm 0.6$ & 12.5 & 18.0 & \\
\hline HD 116121 & 306.5 & -0.4 & $09.5 \mathrm{~V}$ & 9.3 & & $3.3 \pm 0.8$ & 19.0 & 9.8 & \\
\hline HD 116403 & 307.3 & 1.6 & B5 II-III & 9.0 & & $3.0 \pm 0.7$ & 10.7 & 23.0 & \\
\hline HD 116864 & 307.0 & -0.6 & B9 II-III & 9.1 & & $2.4 \pm 0.5$ & - & - & \\
\hline
\end{tabular}

Notes. ${ }^{(1)}$ Corti \& Orellana (in prep.). ${ }^{(2)}$ Humphreys (1978). ${ }^{(3)}$ Obtained of the SIMBAD Astronomical Database (CDS).

rather than the mean value needed for long-term extrapolation. The Hipparcos catalogue quotes the components of the proper motion $\left(\mu_{\alpha} \cos \delta, \mu_{\delta}\right)=(-5.35 \pm 0.59,-2.23 \pm 0.68)$ (Perryman \& ESA 1997).

b) The reliability of the components of the proper motions of WR $48\left(\mu_{\alpha} \cos \delta, \mu_{\delta}\right)=(-1.9 \pm 1.3,19.0 \pm 1.3)$ (Hog et al. 2000) quoted by the Tycho- 2 catalogue could be evaluated in the light of the so-called goodness of the fit, which is given by a quality flag parameter (see Cols. 18 and 19 of Tycho-2 catalogue ${ }^{5}$ ). "This goodness of fit is the ratio of the scatter-based and the model-based error", and may get values

5 http://webviz.u-strasbg.fr/viz-bin/VizieR?-source=I $\% 2 \mathrm{~F} 259$ 
ranging from 0 to 9 . A larger value should be taken as a warning about the proper motion quality. For WR 48, the proper motion in declination has a quality flag of 7.5.

c) Proper motions retrieved from the Position and Proper Motion Extended catalogue (PPMX; Röser et al. 2008) having a problem with the least square fit used to derive the proper motion of a given star were signaled with a flag "P". Those quoted for WR $48\left(\mu_{\alpha} \cos \delta, \mu_{\delta}\right)=(-0.3 \pm 1.7,16.6 \pm$ 1.8) have such a flag.

\subsubsection{Cen OB1}

The stellar association Cen OB1 was first reckoned as such based on spectrophotometric study of Humphreys (1978). A total of 29 stars were listed as members of this association (see Table 8 of Humphreys 1978). The distance modulus derived by Humphreys (1978) for this association is $11.9 \mathrm{mag}(\sim 2.4 \mathrm{kpc})$. Later on, based on a photometric work of Humphreys \& McElroy (1984), the members of Cen OB1 were increased to 106 stars.

For a long time, stellar proper motions have been used to establish the likelihood of membership of a given star in a given stellar grouping, such as an open cluster or an OB-association (Dias et al. 2002; de Zeeuw et al. 1999, and references therein). Today, the availability of proper motion catalogues containing thousands of measurements of stars (e.g. Hipparcos 1997; Tycho2 2000; PPMX 2008; ASCC2.5 2009) distributed all over the sky offers the chance of routinely using proper motionsbased techniques in order to improve our knowledge about the number of stars that may be related to an already known stellar grouping.

Since, according to McClure-Griffiths et al. (2001), GS305+01-24 was "formed by the stellar winds in the Centaurus OB1 association", it is worth making an identification of its members as likely as possible.

To identify the members of Cen OB1 based on the analysis of existing large-scale proper motion catalogues, a two-step procedure was followed. Firstly, the technique of Orellana et al. (2010) was applied to the Hipparcos Catalogue, i.e. down to a visual $(V)$ magnitude ranging from 7.3 to 9.0 (depending on spectral type), on a circular area of radius $7^{\circ}$ centred at $(l, b)=\left(305^{\circ},+0^{\circ}\right)$. The reason for selecting such a large search radius is twofold: $i$ ) to be able to search for star candidates to be members of Cen OB1 on an angular area larger than the one covered by the stars quoted by Humphreys (1978) as members of the association; ii) to include all stars located within a solid angle comparable to, or slightly larger, than the region covered by GS305+0124 (McClure-Griffiths et al. 2001). Bearing in mind that the short time interval covered by the HiPPARcos mission conspires against the accuracy of its quoted proper motions, we applied the same technique in a second step to an square area of $7^{\circ}$ in size of the Tycho- 2 catalogue. This area is also centred at $(l, b)=\left(305^{\circ},+0^{\circ}\right)$. Because the Tycho- 2 catalogue is $90 \%$ complete down to a $V$ magnitude $V=11.5$, we should be able to identify all supergiant stars, regardless of their spectral type, that may belong to Cen OB1, and all main sequence and giant stars whose spectral types are earlier than B 3 V and B 6 III, respectively. In this way, 105 stars were identified as possible members of Cen OB1.

Restricting the above sample only to those stars having both a spectral type classification and photometric data, and retaining from this sub-sample only those stars whose distance lies within $\pm 1 \sigma$ of the mean distance, a total of 54 stars were identified as likely candidates to be members of Cen OB1. They are listed in Table 2. The mean spectrophotometric distance of this grouping turns out to be $2.6 \pm 0.4 \mathrm{kpc}$, in good agreement with previous Cen OB1 distance estimates (Humphreys 1978; Humphreys \& McElroy 1984; Kaltcheva \& Georgiev 1994). Comparing the list of 106 stars of Humphreys \& McElroy (1984) with our listing of 54 stars (see Table 2), only $\sim 24 \%$ of the stars are found to be common to both sets. In the same sense, only ten out of the 22 stars originally listed by Humphreys (1978) as members of Cen OB1 are present in our sample.

To be considered as a possible member of Cen OB1, a given star must have a membership probability $\left(P_{a}\right)$ higher than or equal to 0.5 , according to the Bayesian criterion. This means that if $\Phi_{a}$ represents the spatial function distribution of all stars in the region belonging to the association and $\Phi_{f}$ represents the spatial distribution of all non-member stars present in the same region (see Sect. 4.1 of Orellana et al. 2010), then

$$
P_{a}=\frac{\Phi_{a}}{\Phi_{f}+\Phi_{a}}
$$

the components of the mean proper motion of Cen OB1 stars are $\left(\mu_{\alpha} \cos \delta, \mu_{\delta}\right)=(-4.65 \pm 0.15,-0.92 \pm 0.12)$.

A comparison of this proper motion with the one adopted for WR 48 indicates that whilst the latter is moving from lower galactic latitudes towards the galactic plane, the association Cen OB1, as a whole, is moving in the other way. Therefore, WR 48 is unlikely to belong to this association.

\subsection{One single large $\mathrm{HI}$ structure?}

Relying on the central radial velocity $\left(V_{\mathrm{o}}=-24 \mathrm{~km} \mathrm{~s}^{-1}\right)$ and the expansion velocity $\left(V_{\exp } \sim 7 \mathrm{~km} \mathrm{~s}^{-1}\right)$ of GSH305+01-24 derived by McClure-Griffiths et al. (2001), the Hi brightness temperature distribution covering the velocity range from -40 to $-10 \mathrm{~km} \mathrm{~s}^{-1}$ was re-analysed within the region delimited by $290^{\circ} \leq l \leq 320^{\circ}$ and $-10^{\circ} \leq b \leq+10^{\circ}$ using the LAB Hi survey Kalberla et al. (2005). Though this survey has a lower angular resolution $\left(\mathrm{HPBW}=34^{\prime}\right)$ than other available databases (e.g. Kalberla et al. 2005, HPBW = 14.'4), the difference in angular resolution plays only a minor role when the object under study, like the one we are dealing with, has angular dimensions of several degrees. The main observational characteristics of this distribution are shown in Fig. 1, where a mosaic of six Hr images spanning the velocity from -32 to $-17 \mathrm{~km} \mathrm{~s}^{-1}$ is shown.

Before describing the $\mathrm{Hr}$ images, a word of caution is in place. Considering that toward the inner part of the Galaxy the radial velocity-to-distance transformation is double-valued, the straightforward interpretation that the Hi minima observed, for example, in Fig. 1c represent different structures may not be correct, because in a given radial, velocity range, Hi emission arises from two different locations along the line of sight. Therefore, a large single Hi minima extending both above and below the galactic plane will always appear bisected at lower galactic latitudes by a strong band of galactic Hi emission, giving the impression of two different minima, when in reality it is a single structure. We now describe from a morphological point of view, the main characteristics of the Hi emission observed along the velocity range -32 to $-17 \mathrm{~km} \mathrm{~s}^{-1}$. The images at $-26 \mathrm{~km} \mathrm{~s}^{-1}$ (Fig. 1c), $-23 \mathrm{~km} \mathrm{~s}^{-1}$ (Fig. 1d), and $-20 \mathrm{~km} \mathrm{~s}^{-1}$ (Fig. 1e) of the Hi distribution shows the presence of two welldeveloped Hi minima in the brightness temperature distribution. The one located at positive galactic latitudes (labelled Feature A from here onwards) roughly centred at $(l, b)=\left(305^{\circ},+4^{\circ}\right)$ is ovoidal in shape $(\Delta l, \Delta b) \sim\left(10^{\circ}, 6^{\circ}\right)$ and has its major axis almost parallel to the galactic plane. The minimum located below 

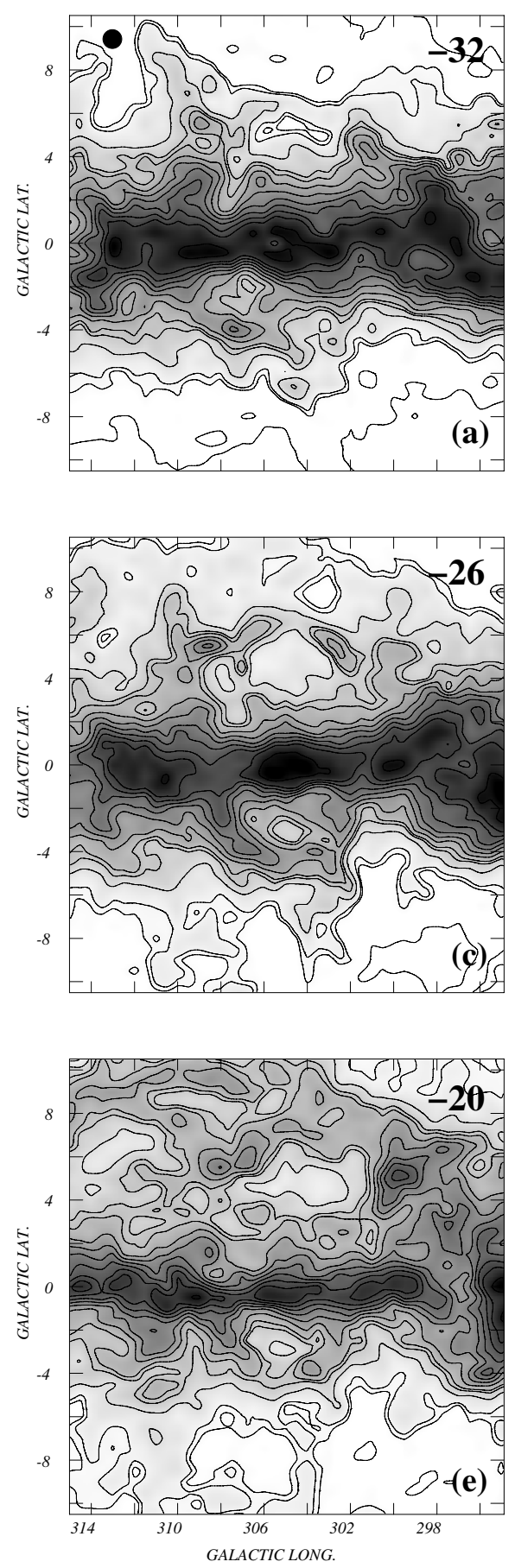
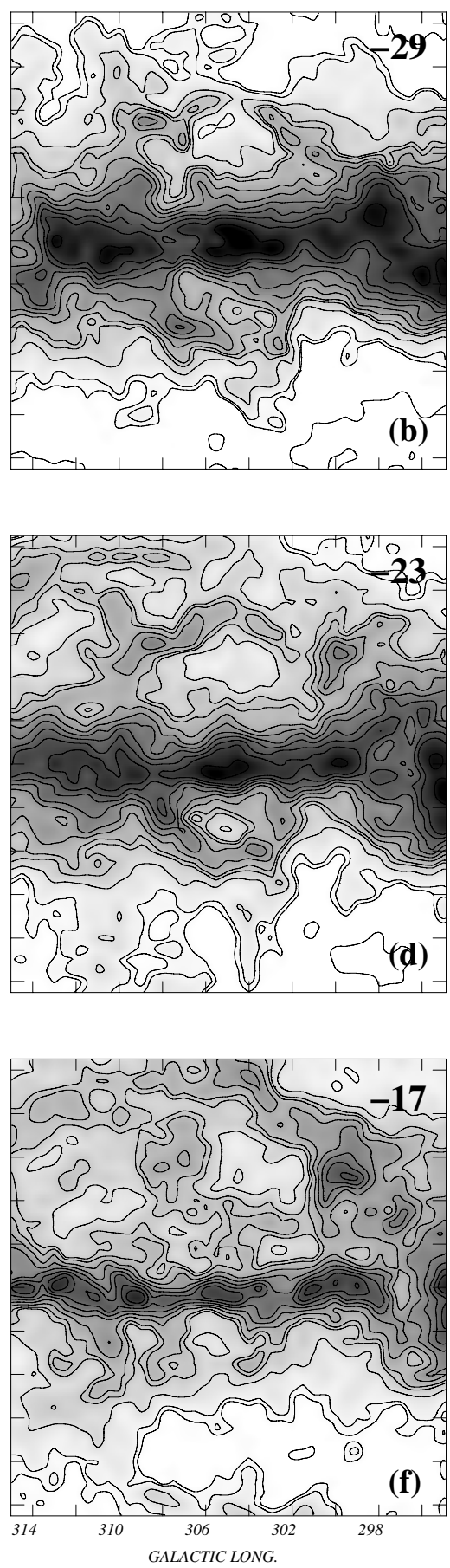

Fig. 1. Mosaic of Hi mean brightness temperature in selected velocity ranges within -33 to $-16 \mathrm{~km} \mathrm{~s}^{-1}$. Each image is a mean of three individual images. The central velocity of each image is indicated at the inner top right-hand corner. The filled circle drawn in the upper lefthand corner of the $\mathrm{HI}$ image at $-32 \mathrm{~km} \mathrm{~s}^{-1}$ indicates the angular resolution of the $\mathrm{HI}$ data. The lowest and highest contours are $15 \mathrm{~K}$ and $105 \mathrm{~K}$, respectively, while the contour spacing is $5 \mathrm{~K}$ until $50 \mathrm{~K}$ and $15 \mathrm{~K}$ from there onwards. The dark grey areas represent regions with high Hi emissivity. the galactic plane (Feature B from here onwards), also elliptical in shape, is centred at $(l, b)=\left(305^{\circ},-3^{\circ}\right)$ and has its major axis along a position angle of $\sim 60^{\circ}$. Position angles are measured counterclockwise from the north galactic pole direction. At more negative radial velocities than those mentioned above (see Fig. 1), Feature A remains visible till $-32 /-30 \mathrm{~km} \mathrm{~s}^{-1}$, where it begins to become undetectable against the overall galactic $\mathrm{HI}_{\mathrm{r}}$ emission at those velocities. On the other hand, Feature B is hardly seen at $-32 \mathrm{~km} \mathrm{~s}^{-1}$ (Fig. 1a). The noticeable Hi minimum seen at $(l, b)=\left(307^{\circ},-2^{\circ} .5\right)$ should not be mistaken with Feature B, because the former, first detected at $-29 /-28 \mathrm{~km} \mathrm{~s}^{-1}$, is located closer to the galactic plane and towards increasing galactic longitudes than Feature B. At radial velocities more positive than $-23 \mathrm{~km} \mathrm{~s}^{-1}$, though both $\mathrm{HI}$ minima are easily identifiable in the $\mathrm{HI}_{\mathrm{I}}$ distribution at $-20 \mathrm{~km} \mathrm{~s}^{-1}$, Feature A begins to lose identity at $-17 \mathrm{~km} \mathrm{~s}^{-1}$, whilst at the same velocity Feature B remains clearly visible. From Fig. 1, it thus appears that Feature A and Feature B may not be detectable along the same velocity interval. In order to further analyse this point, radial velocity versus galactic longitude images were constructed at specific intervals of galactic latitude. In Fig. 2, two such images are shown. The upper panel depicts the mean Hi emission distribution at $3.5 \leq b \leq 4^{\circ}$. 0 , whilst the lower one corresponds to the galatic latitude interval $-3^{\circ} .0 \leq b \leq-2.5$. In both panels, the local Hi emission is depicted by the almost straight ridge of emission seen at both slightly positive (upper panel) and slightly negative velocities (lower panel). The minimum of Hi emission corresponding to either Feature A or Feature B is encircled in both panels by thick dashed figures for easier identification. Admittedly, the above argument is only a qualitative 

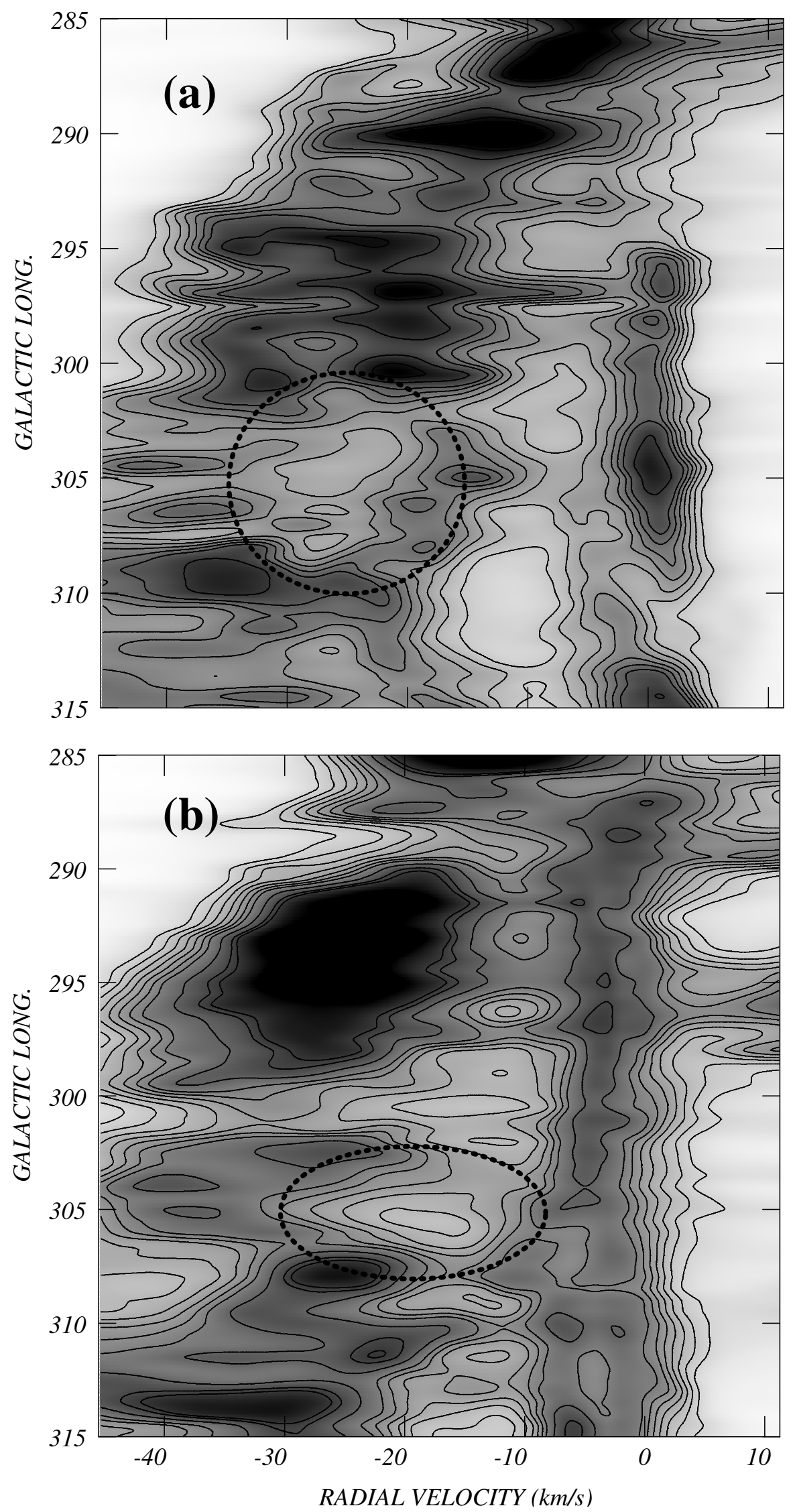

Fig. 2. Velocity-galactic longitude diagram of the mean brightness temperature distribution in two different ranges of galactic latitude. The upper panel shows the velocity-galactic longitude diagram for $3.5 \leq b \leq 4.0$. The dotted circumference signals the location of Feature A (see text). The lower panel shows the same diagram for the galatic latitude strip $-3^{\circ} .0 \leq b \leq-2.5$. The dotted ellipse marks the location of Feature B. one. In Fig. 3, the normalized mean brightness temperature as a function of radial velocity, as derived from Fig. 2 for different galactic longitude ranges, is shown. The continous line in Fig. 3 shows the normalized mean brightness temperature for the range $298^{\circ} \leq l \leq 312^{\circ}$ of Fig. 2 a, whilst the dot-dashed line shows the behaviour observed for Feature B in Fig. 2b. In this case, the interval used to construct the normalized Hi profile is $300^{\circ} \leq l \leq 310^{\circ}$. The normalized emission was computed by 


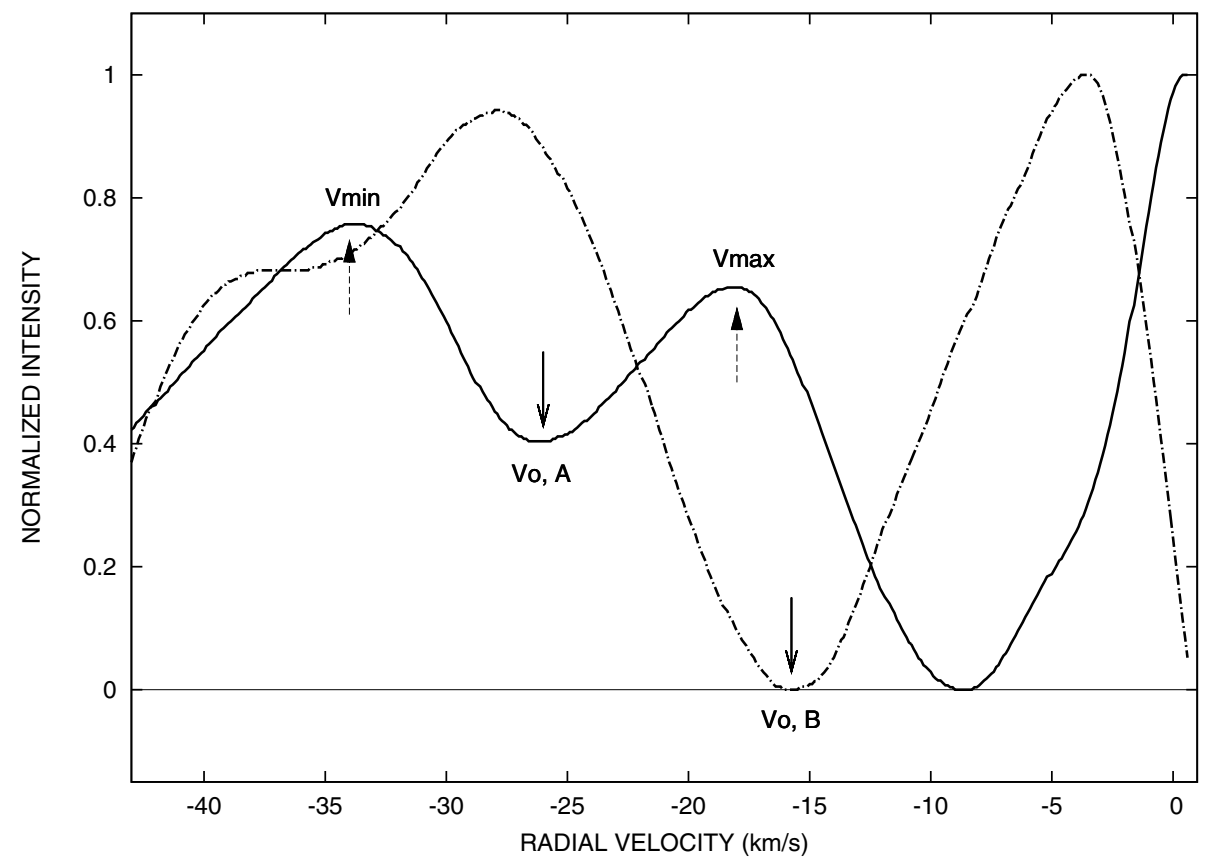

Fig. 3. Normalized mean Hi brightness temperature profiles for the range $298^{\circ} \leq l \leq 312^{\circ}$ (Feature A, continuos line) and between $300^{\circ} \leq$ $l \leq 310^{\circ}$ (Feature B, dash-dotted line profile). The arrows mark the mean radial velocity for Feature $\mathrm{A}\left(V_{\mathrm{o}, \mathrm{A}}\right)$ and Feature $\mathrm{B}\left(V_{\mathrm{o}, \mathrm{B}}\right)$, respectively. substracting from each point the minimum value of the corresponding scan (19 K and $31 \mathrm{~K}$ for Features A and Feature B, respectively) and then dividing by the corresponding maximum (27.2 K and 21.1 K for Features A and B, respectively). Figure 3 clearly shows that the mean radial velocity and the FWHM of both features, namely $V_{\mathrm{o}, \mathrm{A}}=-26 \mathrm{~km} \mathrm{~s}^{-1}$ and $9 \mathrm{~km} \mathrm{~s}^{-1}$, respectively, for Feature $\mathrm{A}$, and $V_{\mathrm{o}, \mathrm{B}}=-16 \mathrm{~km} \mathrm{~s}^{-1}$ and $14 \mathrm{~km} \mathrm{~s}^{-1}$, respectively, are different. This would not be the expected behaviour if both features were part of a single large Hi void, because then one would expect the mean radial velocity of both features (A and B) to be, within errors, similar and the FWHM of Feature B to be smaller than the FWHM of Feature A. Mean radial velocities and FWHM were derived from a Gaussian fitting, and both are accurate to within $\pm 1 \mathrm{~km} \mathrm{~s}^{-1}$.

Using the linear fit and the power law fit of the galactic rotation model of Fich et al. (1989), the kinematic distances of both $\mathrm{HI}_{\mathrm{I}}$ structures turned out to be $2.5 \pm 0.9 \mathrm{kpc}$ (Feature A) and $1.7 \pm 0.7 \mathrm{kpc}$ (Feature B). These distances are a mean weighted value of the individual distances provided by the different models. The weight of the individual distance determinations stems from the assumption of an uncertainty of $\pm 8 \mathrm{~km} \mathrm{~s}^{-1}$ due to non-circular motions (Burton 1988).

Therefore, under the assumption that both shells have a lineof-sight dimension comparable to those observed in the plane of the sky, and relying exclusively on the derived mean distances for both $\mathrm{H}_{\mathrm{I}}$ features, the line-of-sight width of both shells would be insufficient to overlap them in space, even within the large errors quoted for the individual distances of both features.

It is thus believed that Feature A and Feature B are unrelated Hi structures and not different manifestations of the same feature (GSH305+01-24), as put forward by McClure-Griffiths et al. (2001). In this context, it is worth mentioning that Feature B was interpreted (Cappa de Nicolau \& Niemela 1984) as being an Hi feature exclusively associated with the Wolf-Rayet

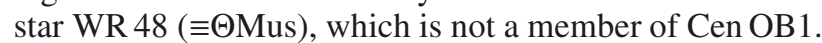

The mean brightness temperature distribution in the range -26 to $-19 \mathrm{~km} \mathrm{~s}^{-1}$ is presented in Fig. 4. The shell GSH305+01-24 reported by McClure-Griffiths et al. (2001) is shown as a thick dark dotted ellipse, whilst the approximate boundaries of Feature A are depicted by a light dotted line.
Following the standard nomenclature, Feature A from here onwards will be referred to as GS305+04-26. The high-latitude border of this structure has a mean brightness temperature of $20 \mathrm{~K}$ above the surrounding background galactic emission, a fact that favours its clear identification. Unfortunately, the lowlatitude extreme of GS305+04-26 is not detected because it is projected onto the strong $\left(T_{b} \sim 90 \mathrm{~K}\right)$ emission arising from the $\mathrm{HI}$ located at the far kinematic distance $(\sim 7.5 \mathrm{kpc})$ along this line of sight, making the effort of attempting to disentangle it from the overall galactic Hi emission a pointless task. The physical parameters of GS305+04-26 are derived from a least square fit of an ellipse to the Hi peaks defining the shape of GS305+04-26 above $b=2^{\circ}$. From this fit, shown in Fig. 4 by the thin dotted line, the supershell centre is $(l, b)=\left(305^{\circ},+4^{\circ}\right)$ and the major and minor angular semi-axes of GS305+04-26 are $\sim 5^{\circ}$ and $3^{\circ}$, respectively. The fitting errors of the supershell centre are $\pm 00^{\circ}$. At the distance of GS305+04-26, these angular dimensions are equivalent to $220 \pm 70\left(R_{\max }\right)$ and $135 \pm$ $40\left(R_{\min }\right)$ pc, respectively.

Under the assumption of an optically thin Hr emission, the total Hi mass of a structure located at a distance $d(\mathrm{kpc})$ that subtends a solid angle $\Omega$ (squared arc min) is given by

$M_{\mathrm{HI}}=0.0013 d^{2} \Delta V \triangle T_{\mathrm{B}} \Omega\left(M_{\odot}\right)$,

where $\Delta V$ is the velocity interval covered by the structure, expressed in $\mathrm{km} \mathrm{s}^{-1}$, and $\Delta T_{\mathrm{B}}(\mathrm{K})$ is the mean brightness temperature of the structure defined as $\Delta T_{\mathrm{B}}=\left|T_{\mathrm{sh}}-T_{\mathrm{bg}}\right|$, where $T_{\mathrm{sh}}$ refers to the mean shell $\mathrm{HI}$ brightness temperature and $T_{\mathrm{bg}}$ is the brightness temperature defining the outer level of the Hi structure. In this way, the Hi mass of GS305+04-26 is of the order of $M_{\mathrm{HI}}=(1.8 \pm 0.4) \times 10^{5} M_{\odot}$. Assuming a helium abundance of $34 \%$ by mass, the total gaseous mass of GS305+04-26 is about $M_{\mathrm{t}}=(2.4 \pm 0.5) \times 10^{5} M_{\odot}$.

The expansion velocity plays a key role (along with the shell's total mass) at the time of evaluating the shell's total kinetic energy. One way of determining this parameter stems from the use of position-position $\mathrm{HI}_{\mathrm{i}}$ images (e.g. those shown in Fig. 1) of the feature under study. In this case, the expansion velocity $\left(V_{\text {exp }}\right)$ is determined using the maximum approaching $\left(V_{\max }\right)$ and maximum receding $\left(V_{\min }\right)$ radial velocities of 


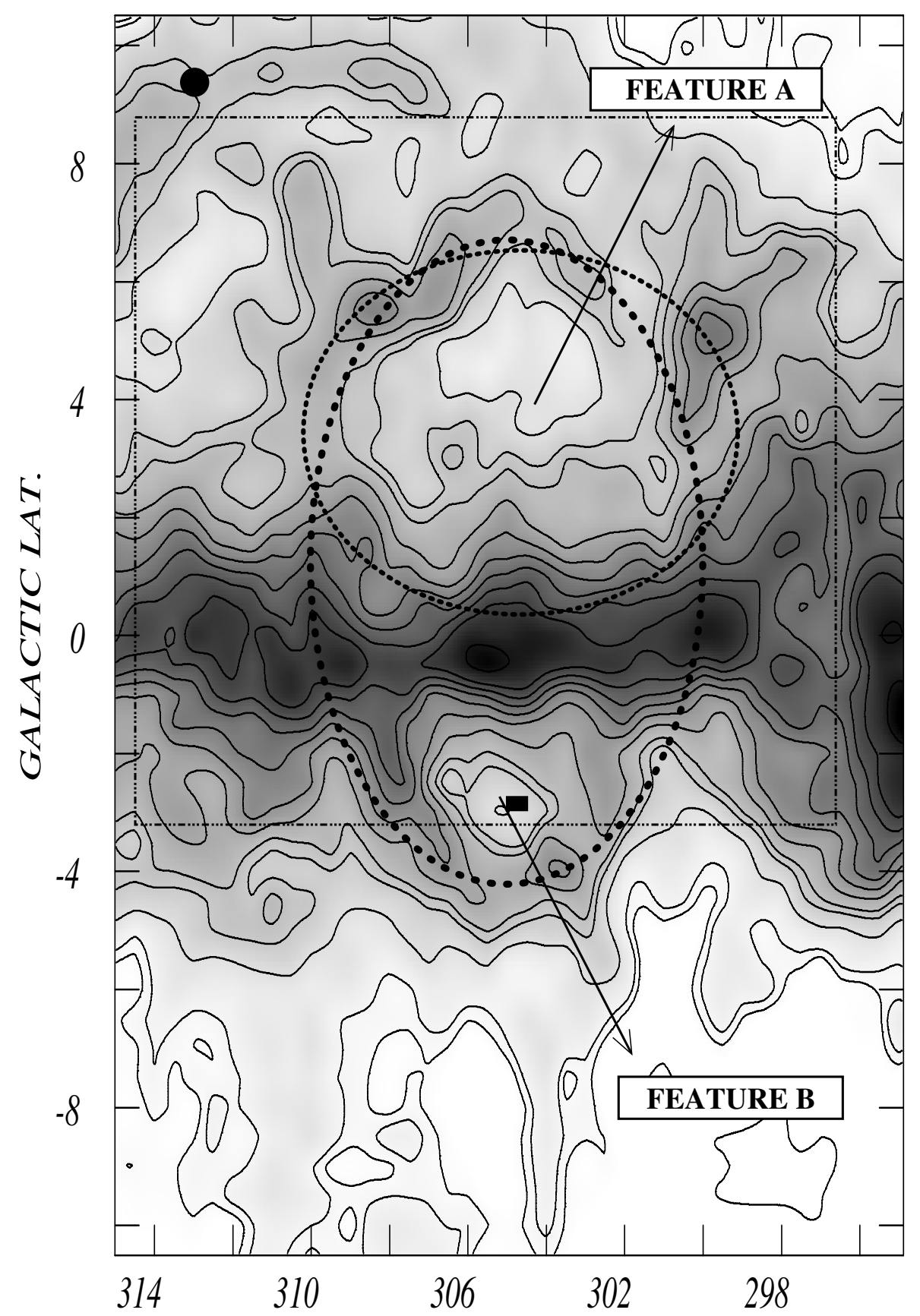

GALACTIC LONG.
Fig. 4. Grey-scale representation of the mean Hi brightness temperature in the velocity range -26 to $-19 \mathrm{~km} \mathrm{~s}^{-1}$. The light dot ellipse represents the least square fit to the Hi peaks defining GS305+04-26. The location of GSH305+01-24 (McClure-Griffiths et al. 2001) is depicted by a dark dot ellipse. The dash-dotted rectangle delimits the region shown in Fig. 5. The small black rectangle shows the position of the star WR 48, $(l, b)=$ $(304.7,-2.5)$. The angular resolution of these data is given by the filled dot drawn in the upper left-hand corner. those $\mathrm{H}_{\mathrm{I}}$ features identified as belonging to the expanding structure. Applying this procedure, an expansion velocity $V_{\exp }=$ $0.5 \times\left(V_{\max }-V_{\min }\right)$ of $\sim 6 \mathrm{~km} \mathrm{~s}^{-1}$ is derived. Generally speaking, $V_{\text {exp }}$ determined in this way is a lower limit to the real expansion velocity, because "extreme" velocity gas associated with the expanding Hi feature could be missed owing to confusion effects with the overall galactic Hi emission. Another way of determining $V_{\text {exp }}$ is based on the use of velocity-position (either galactic latitude or galactic longitude) diagrams. To evaluate $V_{\exp }$, the normalized Hi brightness temperature profile shown in Fig. 3 (continuous line) is used. By making a Gaussian fit to the HI emission peaks, $V_{\max }$ and $V_{\min }$ can be derived along with $V_{\mathrm{o}, \mathrm{A}}$, which corresponds to the velocity between $V_{\max }$ and $V_{\min }$, where the minimum value of the $\mathrm{HI}_{\mathrm{I}}$ emission is observed. We derived $V_{\mathrm{o}, \mathrm{A}}=-26 \pm 1 \mathrm{~km} \mathrm{~s}^{-1}$. The approaching gas is observed at $V_{\text {min }}=-34 \mathrm{~km} \mathrm{~s}^{-1}$ and the receding gas at $V_{\max }=-17 \mathrm{~km} \mathrm{~s}^{-1}$. Therefore, the velocity extent of the observed H I emission associated with GS305+04-26 is consistent with an expansion velocity of $V_{\exp } \simeq 8 \pm 1 \mathrm{~km} \mathrm{~s}^{-1}$. Adopting this value for $V_{\exp }$ and using the total mass calculated for GS 305+04-26, the kinetic energy $\left(E_{\mathrm{k}}=0.5 M V_{\exp }^{2}\right)$ of the expanding structure is $E_{\mathrm{k}} \sim(1.7 \pm 0.4) \times 10^{50} \mathrm{erg}$.

The dynamic $\left(t_{\mathrm{dyn}}\right)$ age of GS305+04-26 was derived by adopting the Weaver et al. (1977) analytic solutions for a thin expanding shell created by a continuous injection of mechanical energy. Under this assumption, the dynamic age is given by

$t_{\text {dyn }}=0.55 \times 10^{6} R_{\mathrm{s}} / V_{\exp }(\mathrm{yr})$, 
where $R_{\mathrm{S}}$ is the radius of the structure, expressed in units of pc. The expansion velocity is given in units of $\mathrm{km} \mathrm{s}^{-1}$, and the constant in Eq. (3) represents the mean value between the energyand momentum-conserving cases. Adopting the harmonic mean of the major and minor semi-axes for $R_{\mathrm{s}}=\sqrt{\left(R_{\max } \times R_{\min }\right)}$, and using an expansion velocity of $8 \mathrm{~km} \mathrm{~s}^{-1}$, a $t_{\mathrm{dyn}} \sim 10^{7} \mathrm{yr}$ is derived.

\section{Discussion}

\subsection{Origin of GS $305+04-26$}

Could GS 305+04-26 have been created by the stellar winds and supernova explosions of the most massive members of Cen OB1? Under the assumption that all stars listed in Table 2 are actually members of Cen OB1 and using a very simple geometrical model, we shall attempt to answer this question. In our model, we shall also assume that all the stars belonging to Cen OB 1 were at the beginning closely packed at the centroid of GS 305+04-26.

The total mechanical energy $\left(E_{\mathrm{w}}\right)$ injected by a star along its lifetime is given by $E_{\mathrm{W}}=L_{\mathrm{w}} \tau$, where $L_{\mathrm{w}}$ is the stellar mechanical luminosity $\left(L_{\mathrm{w}}=0.5 \dot{M} v_{\infty}^{2}\right)$ and $\tau$ is the age of the star. Under the assumption of solar metallicity, the stellar mass loss rate $(\dot{M})$ and the wind terminal velocity $\left(v_{\infty}\right)$, were derived from de Jager et al. (1988),

$\log \dot{M}=1.769 \log L / L_{\odot}-1.676 \log T_{\text {eff }}-8.158$

$\log v_{\infty}=1.23-0.3 \log L / L_{\odot}+0.55 \log M / M_{\odot}+0.64 \log T_{\text {eff }}$,

where $L$ is the stellar luminosity expressed in solar luminosity units and $T_{\text {eff }}$ is the effective stellar temperature expressed in $\mathrm{K}$.

In Eq. (5), $M$ indicates the mass of the star (in solar mass units). The value of $v_{\infty}$ agrees with observations to within $20 \%$. For a given spectral type, the basic stellar parameters $(L, M$ and $T_{\text {eff }}$ ) were adopted from the values given by Schmidt-Kaler (Th. 1982).

To provide an estimate for the stellar mass using both the spectral type given in the fourth column of Table 2 and the relationship between stellar mass and effective temperature given by Schmidt-Kaler (Th. 1982), a mass estimate (eighth column of Table 2) was derived for every star. Following Schaller et al. (1992), the age $(\tau)$ is derived. For stars belonging to the main sequence, an age estimate was drawn under the assumption that they are in the nuclear hydrogen burning phase, whilst the ages for the evolved stars (those having luminosity classes other than $V$ ) were determined after adding together the time spent in the hydrogen, helium and carbon nuclear burning phases. The stellar ages are given in the ninth column of Table 2. Following the above procedure, late B-type stars with uncertain spectral classification (HD 109253, HD 110912 , HD 111121, and HD 116864) have masses, and hence stellar ages, which are quite uncertain. Consequently, neither $M$ nor $\tau$ was derived for these four stars. The total mechanical energy injected by the stars of Cen OB1 is the sum of the contributions of all stars along their lifetime. Based on the above, the total mechanical energy input by the stars of Cen OB1 into its local ISM is $(2.7 \pm 0.1) \times 10^{51} \mathrm{erg}$. Using de Jager et al. (1988) to estimate the wind terminal velocity and the parameterization of the mass-loss rate of Lamers \& Leitherer (1993),

$\log \dot{M}=1.738 \log L / L_{\odot}-1.352 \log T_{\text {eff }}-9.547$, results in a total mechanical energy input lower by almost $30 \%$. Equation (6) is the average relation for $\dot{M}$ in a sample of galatic O stars studied by Lamers \& Leitherer (1993). According to these authors, the standard deviation of individual mass-loss rates is $\sigma=0.23$.

Though theoretical models predict that $20 \%$ of the windinjected energy is converted into the mechanical energy of an expanding shell (Weaver et al. 1977), the energy conversion efficiency seems to be as low as 2-5\% (Cappa et al. 2003) from an observational viewpoint.

Additional sources of energy input may have been present within the boundaries of GS 305+04-26. It is worth pointing out that supernovae explosions $\left(\mathrm{SN}_{\mathrm{e}}\right)$ may have taken place among the most massive members of Cen OB1. Though there are no supernova remnants catalogued within the solid angle covered by GS $305+04-26$, three pulsars located within the boundaries of this shell indicate that $\mathrm{SN}_{\mathrm{e}}$ took placed in the past. The pulsars are PSR J1253-5820 $\left(d=2.2 \mathrm{kpc},(l, b)=\left(303^{\circ} .2\right.\right.$, $+4.5)$ ), PSR J1334-5839 $(d=2.4 \mathrm{kpc},(l, b)=(308.5,+3.7))$, and PSR J1254-6150 $\left(d=2.24 \mathrm{kpc},(l, b)=\left(303^{\circ} .3,+1^{\circ} .02\right)\right)$. The characteristic age of the pulsars $\left(\tau_{\mathrm{c}}\right)$ are $\sim 2 \times 10^{6} \mathrm{yr}$ (PSR J1253-5820), $\sim 5 \times 10^{6}$ yr (PSR J1254-6150), and $\sim 8 \times$ $10^{7}$ yr (PSR J1334-5839), respectively (Manchester et al. 2005). The distance of PSR J1253-5820 has been taken from Noutsos et al. (2008), whilst the distance of the other two is given by Manchester et al. (2005).

From Table 2, there is evidence of continuous star formation, because the stellar ages $\tau$ covered the range from $\sim 5$ to $24 \mathrm{Myr}$, with a mean value of $12.6 \pm 5$ Myr. Bearing in mind the fact of a continuous star formation, we shall adopt as the age of the association the age of the oldest stars likely to be members of Cen OB1. Hence, Cen OB1 would be $\sim 2 \times 10^{7}$ years old. The ages of PSR J1253-5820 and PSR J1254-6150 are consistent with the assumption that they could have been born in $\mathrm{SN}_{\mathrm{e}}$ of stars members of Cen OB1. On the other hand, PSR J1334-5839 has an age almost an order of magnitude greater that the age of Cen OB1. Thus, the massive progenitor star of this pulsar very likely was not a member of Cen OB1 and played no role in the formation of GS305+04-26.

Assuming that the progenitors of both pulsars were members of Cen OB1, they may have contributed to the formation of GS305+04-26 in two ways; first, by injecting mechanical energy along their stellar lifetime, and second by their final $\mathrm{SN}_{\mathrm{e}}$. A lower limit to the mechanical energy input by the stellar progenitors of the pulsars prior to their explosion as $\mathrm{SN}_{\mathrm{e}}$ could be derived, assuming that each stellar progenitor spent time as a "stellar" object, equal to the age of the oldest star likely to be a member of Cen OB1 (HD 109937) minus the pulsar's age. In this way, the progenitors of each pulsar could have been main sequence stars in the range from 10 to $12 M_{\odot}$. Using Eqs. (4) and (5), both progenitor stars could have injected extra mechanical energy of $\sim 2.4 \times 10^{51}$ erg during their lives as stars.

The effect of supernovae on wind-driven bubble evolution may be similar to that of stellar winds acting as an input energy source (McCray \& Kafatos 1987). These authors show that assuming a standard initial mass function for a stellar association having $N_{*}$ stars with mass greater that $7 M_{\odot}$, the mean energy input by $\mathrm{SN}_{\mathrm{e}}$ is given by $E_{\mathrm{SN}} \sim 2.0 \times$ $10^{49} N_{*} E_{51} \tau_{\mathrm{OB}}$ erg, where $E_{51}$ is the supernova explosion expressed in units of $10^{51} \mathrm{erg}$ and $\tau_{\mathrm{OB}}$ is the association's age given in units of $10^{6} \mathrm{yr}$. Inserting $N_{*}=2$ and $\tau_{\mathrm{OB}} \simeq 24 \mathrm{Myr}$, we obtain $E_{\mathrm{SN}} \sim 10^{51} \mathrm{erg}$. Certainly, the assumption that only two $\mathrm{SN}_{\mathrm{e}}$ (those that originated the pulsars we are observing nowdays) took place among the stars of Cen OB1 must be viewed as 


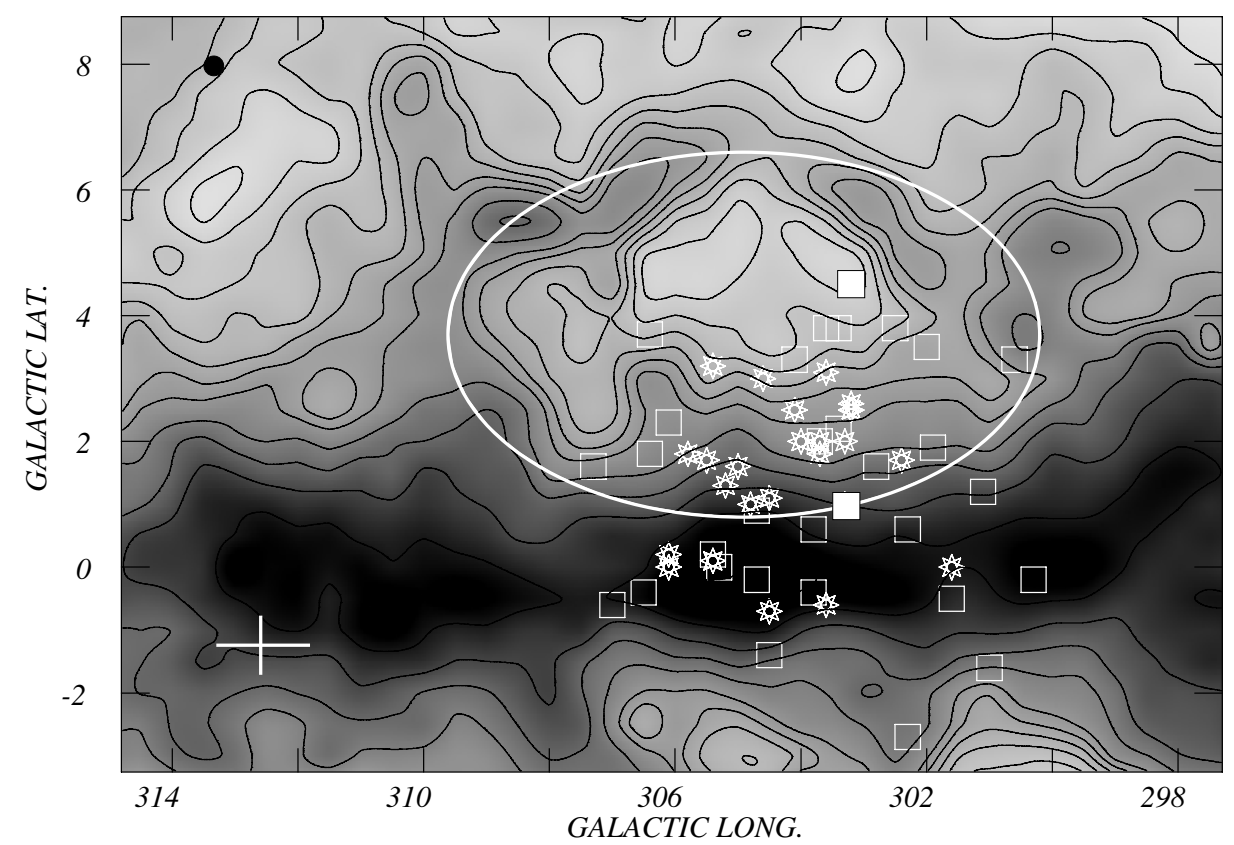

Fig. 5. Blowup of the region delimited by a dash-dot rectangle in Fig. 4. The angular resolution of these data is given by the filled dot drawn in the upper left-hand corner of the image. The white thin line ellipse represents GS305+04-26. The white boxes mark the location of the pulsars. The star symbol indicates the location of Cen OB1 members identified as such by both Humphreys \& McElroy (1984) and Corti \& Orellana (in prep.), whilst the unfilled white squares signal the position of those stars considered to be members of Cen OB1 only by Corti \& Orellana (in prep.).

a gross simplification, because in the past more stars of Cen OB1 could have gone through the $\mathrm{SN}_{\mathrm{e}}$ phase without leaving observable evidence that those events did happen. If the end stellar product of those hypothetical explosions were pulsars, they could be there and at the same time remain undetected due to their highly anisotropic emission. As a result, by assuming that only two $\mathrm{SN}_{\mathrm{e}}$ took place, a lower limit is provided to the total energy input in this way to the ISM local to Cen OB1.

Therefore, adding up the energy injected into the ISM by the stellar winds of the stars of Cen OB1 and the progenitor stars of both pulsars and the energy injected by both $\mathrm{SN}_{\mathrm{e}}$, a total of $(3.6 \pm 0.1) \times 10^{51}$ erg may have been injected into the ISM by the stars of Cen OB1 along their lives. In this case, the kinetic energy of GS305+04-26 would imply an energy conversion efficiency of $\sim 3 \%$ in very good agreement with the results of Cappa et al. (2003).

\subsection{Explaining the off-centre location of Cen OB1}

In the ideal case of a group of massive stars being at rest with respect to its local ISM, it is expected that this group will be located at the centre of a hypothetical stellar-wind bubble blown by this group of massive stars. If the stars are not at rest with respect to their surroundings, they will likely occupy an eccentric location with respect to the bubble's centre. Figure 5 clearly shows that most of the Cen OB1 stars are located towards the low-latitude and low-longitude sector of GS305+04-26. In order to explain, this location, in general terms, we shall follow the procedure outlined by van der Sluys \& Lamers (2003) (after correcting the sign of the $w_{\odot}$ term in their Eq. (19)) and Cichowolski et al. (2008).

Using the mean proper motions of Cen OB1 $\left(\left(\mu_{\alpha} \cos \delta, \mu_{\delta}\right)=\right.$ $(-4.65 \pm 0.15,-0.92 \pm 0.12)$.), a radial velocity of $-26 \pm 1 \mathrm{~km} \mathrm{~s}^{-1}$ and a distance of $2.5 \pm 0.9 \mathrm{kpc}$ (radial velocity and distance of GS305+04-26) as the original distance of Cen OB1, the stellar peculiar velocity components are

$$
\begin{gathered}
v_{\mathrm{pec}, r}=-2.2 \pm 14.8 \mathrm{~km} \mathrm{~s}^{-1}, \\
v_{\mathrm{pec}, l}=12.4 \pm 30.8 \mathrm{~km} \mathrm{~s}^{-1}, \\
v_{\mathrm{pec}, b}=-15.6 \pm 4.1 \mathrm{~km} \mathrm{~s}^{-1},
\end{gathered}
$$

where $v_{\text {pec }, r}$, is the peculiar radial velocity, $v_{\text {pec }, l}$ the peculiar velocity along galactic longitude, and $v_{\text {pec, } b}$ the peculiar velocity along galactic latitude. The corresponding spatial peculiar velocity of the star,

$$
\begin{aligned}
& v_{\mathrm{pec}}=\sqrt{v_{\mathrm{pec}, r}^{2}+v_{\mathrm{pec}, l}^{2}+v_{\mathrm{pec}, b}^{2}}, \\
& \text { is } v_{\mathrm{pec}}=20.0 \pm 18.0 \mathrm{~km} \mathrm{~s}^{-1} .
\end{aligned}
$$

According to van der Sluys \& Lamers (2003), only two angles, labelled $i$ and $\phi$, are needed to completely define the spatial orientation with respect to the observer of the structure created by a star moving through the ISM at a high peculiar velocity. The angle $i$ is measured from the line of sight towards the observer $\left(i=90^{\circ}\right.$ corresponds to the plane of the sky), whilst $\phi$ is measured on the plane of the sky, counterclockwise from the tip of the peculiar velocity vector (see Fig. 4 of van der Sluys \& Lamers 2003). Following van der Sluys \& Lamers (2003), the above angles are defined as

$$
\begin{aligned}
i & =\arcsin \left(\frac{v_{\mathrm{pec}, r}}{v_{\mathrm{pec}}}\right) \\
\phi & =\arctan \left(\frac{v_{\mathrm{pec}, l}}{v_{\mathrm{pec}, b}}\right)+\varepsilon
\end{aligned}
$$

where $\varepsilon$ is the angle between the line of constant declination and the line of constant galactic latitude at the position of the 


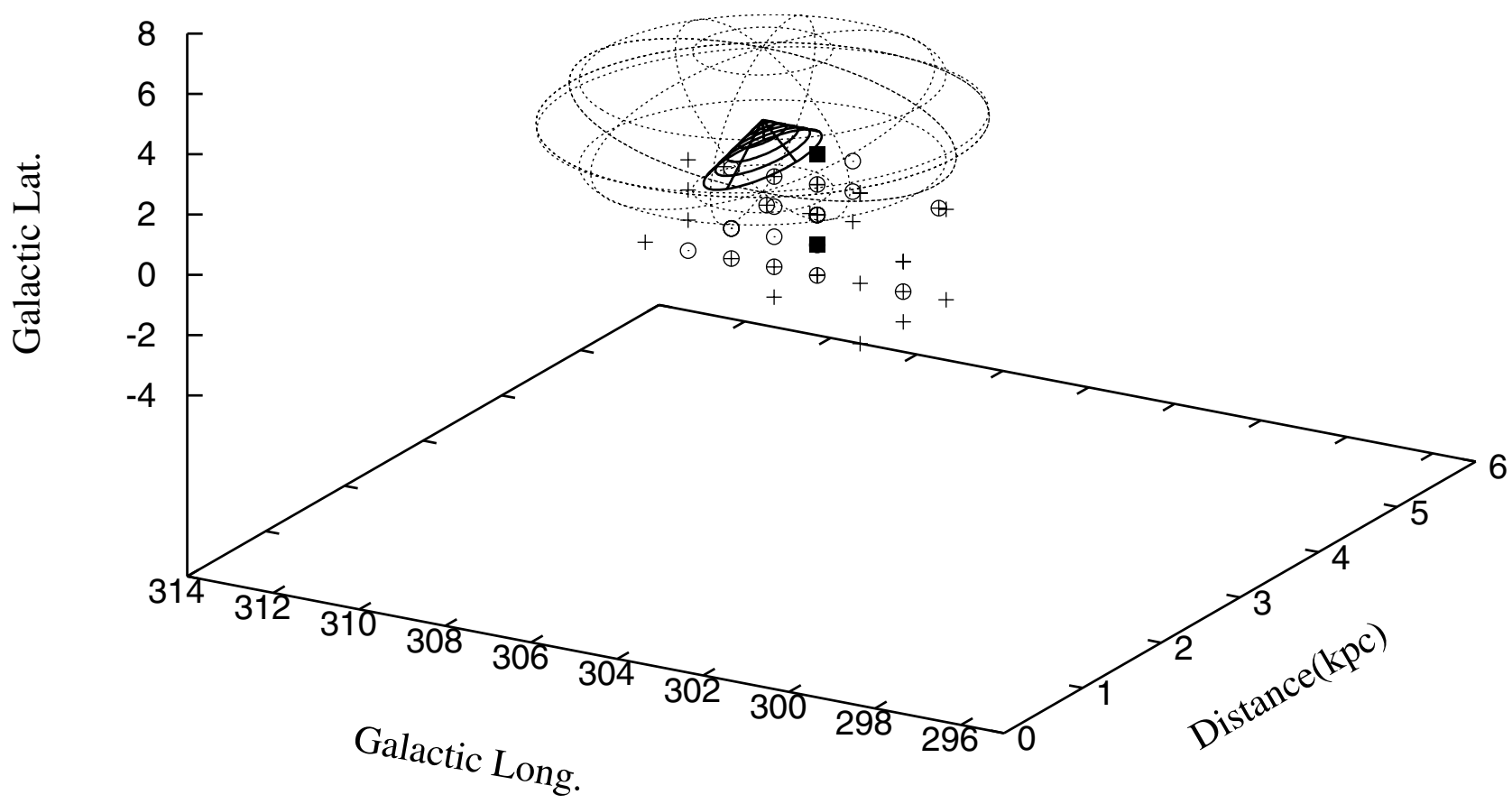

Fig. 6. Three-dimensional sketch of GS305+04-26 and the star members of Cen OB1. The large Hi shell is shown as a dotted ellipsoid. The location of both pulsars is given by black squares. The open circles indicate the location of Cen OB1 members identified as such by Humphreys \& McElroy (1984) and Corti \& Orellana (in prep.) whilst the plus sign marks the position of those stars considered to be members of Cen OB 1 only by Corti $\&$ Orellana (in prep.). The projection of the conical structure refered to in the text is also depicted.

star. In our case $\varepsilon \sim 1^{\circ}$, and the angles are $i=95^{\circ} \pm 45^{\circ}$, and $\phi=140^{\circ} \pm 70^{\circ}$.

At first glance, one may think that all stars of Cen OB1 should be seen projected onto the interior of GS305+04-26. But, a look at Fig. 5, taking into account the uncertainties of the major and minor semi-axes of GS305+04-26 (error bars drawn in the lower left corner), shows that 12 stars are definitively located outside the large Hi shell. Under the assumption that these peculiar velocities are inherent to the process of their formation, the sequence of events put forward by Sancisi (1974) could explain the external position of the stars of Cen OB1 with respect to the expanding HI shell. Certainly, in the case of Cen OB1, stellar winds are the main triggering mechanism of the expansion of GS305+04-26 and not $\mathrm{SN}_{\mathrm{e}}$, as was the case in Per OB2. Though these stars have all the basic ingredients to create around them their own IB (which should be observable as a minimum in the HI brightness temperature distribution towards these stars), these minima are not observed. The lack of detection may be explained by the following facts: $i$ ) the stars in question are seen projected onto the galactic plane where the Hi brightness temperature is high and varies quite rapidly with position; $i i)$ the dual-value radial velocity relationship implies that any Hi minimum related to an IB located at the near kinematic distance will very likely be filled in by $\mathrm{HI}$ emission arising from the far kinematic distance region. Based on the above, IB around individual massive stars will be hard to recognize as such when the stars are seen projected towards the galactic plane and the $21 \mathrm{~cm}$ line emission is observed with a radiotelescope like the one used to produce the LAB Hi all-sky survey.

Summing up, and within their large uncertainties, the bulk peculiar spatial velocity of the stars of Cen OB1 and the angles $i$ and $\phi$ are consistent with the present-day location of Cen OB1 with respect to the centroid of GS305+04-26. The off-centre location of PSR J1253-5820 and PSR J1254-6150 is quite consistent with the peculiar spatial velocities of these objects (Cordes \& Chernoff 1998).

To explain the location of the OB-association projected onto the southernmost border of GS305+04-26, a peculiar velocity of $10 \pm 5 \mathrm{~km} \mathrm{~s}^{-1}$ would be needed along $12.6 \pm 5 \mathrm{Myr}$ (the age of the association). This velocity is, within its large error, consistent with the bulk peculiar motions derived for the stars of Cen OB1. A three-dimensional sketch of the relative location of the stars with respect to GS305+04-26 is given in Fig. 6. There, the supershell is represented by the ellipsoid shown in dotted lines. The large triangle-like symbol depicts the projection of a conical structure whose apex is located at the centre of GS305+04-26, and its bisecting line is oriented along the angles $i$ and $\phi$ derived above. The base of the cone points towards both lower galactic latitudes and longitudes. The aperture of the cone is mostly given by the uncertainty in determining the angle $\phi$.

\section{Conclusions}

Examining the $\mathrm{HI}$ distribution towards the area covered by Cen OB1, in the light of new data that redefine the stellar membership of this association, a new large Hi shell has been found. This structure, designated GS305+04-26, is elliptical in shape with major and minor axes of 440 and $270 \mathrm{pc}$, respectively. The velocity interval where GS305+04-26 is observable spans the range from $\sim-33$ to $\sim-17 \mathrm{~km} \mathrm{~s}^{-1}$. The central velocity is $-26 \pm 1 \mathrm{~km} \mathrm{~s}^{-1}$, and its expansion velocity is about $\sim 8 \mathrm{~km} \mathrm{~s}^{-1}$. The kinematic distance of this large structure is $2.5 \pm 0.9 \mathrm{kpc}$, and has a total gaseous mass of about $M_{\mathrm{t}}=(2.4 \pm 0.5) \times 10^{5} M_{\odot}$.

Evidence has been provided in the sense that the stellar winds of the 54 stars likely to be members of Cen OB1 may well explain the kinetic energy of this large structure. The pulsars PSR J1253-5820 and PSR J1254-6150 have both distances and characteristic ages compatible with the idea that they were 
born as the results of $\mathrm{SN}_{\mathrm{e}}$ undergone in the past by massive members of Cen OB1.

The present-day eccentric location of the stars of Cen OB1 with respect to the centroid of GS305+04-26 can be understood by the peculiar velocities of the stars relative to its local ISM.

Comparing the proper motion of the Wolf-Rayet star WR 48 with the bulk proper motions of Cen OB1, it is highly unlikely that the Wolf-Rayet star can be considered a member of the stellar association.

Acknowledgements. This work was partially supported by Consejo Nacional de Investigaciones Científicas y Técnicas CONICET under projects PIP 01299 and 01359 and by Universidad Nacional de La Plata (UNLP) under projects 11/G091 and 11/G096. M. A. Corti would like to thank Dra E Gularte Scarone for her help in preparing Fig. 6 and Dr. N. Walborn for providing us with updated information of some of the stellar data quoted in Table 2. We would like to thank the referee for her/his constructive remarks.

\section{References}

Burton, W. B. 1988, in Galactic and Extragalactic Radio Astronomy, 2nd edn. (Berlin: Springer-Verlag), 295

Cappa, C. E., Arnal, E. M., Cichowolski, S., Goss, W. M., \& Pineault, S. 2003, in A Massive Star Odyssey: From Main Sequence to Supernova, eds. K. van der Hucht, A. Herrero, \& C. Esteban, IAU Symp., 212, 596 Cappa de Nicolau, C., \& Niemela, V. S. 1984, AJ, 89, 1398

Cichowolski, S., Pineault, S., Arnal, E. M., \& Cappa, C. E. 2008, A\&A, 478, 443

Cordes, J. M., \& Chernoff, D. F. 1998, ApJ, 505, 315

de Jager, C., Nieuwenhuijzen, H., \& van der Hucht, K. A. 1988, A\&AS, 72, 259

de Zeeuw, P. T., Hoogerwerf, R., de Bruijne, J. H. J., Brown, A. G. A., \& Blaauw, A. 1999, AJ, 117, 354

Dias, W. S., Alessi, B. S., Moitinho, A., \& Lépine, J. R. D. 2002, A\&A, 389, 871
Fich, M., Blitz, L., \& Stark, A. A. 1989, ApJ, 342, 272

Heiles, C. 1979, ApJ, 229, 533

Heiles, C. 1984, ApJS, 55, 585

Hog, E., Fabricius, C., Makarov, V. V., et al. 2000, VizieR Online Data Catalog, I/259

Humphreys, R. M. 1978, ApJS, 38, 309

Humphreys, R. M., \& McElroy, D. B. 1984, Vizier Online Data Catalog, V/44

Kalberla, P. M. W., Burton, W. B., Hartmann, D., et al. 2005, A\&A, 440, 775

Kaltcheva, N. T., \& Georgiev, L. N. 1994, MNRAS, 269, 289

Kharchenko, N. V. 2001, Kinematika i Fizika Nebesnykh Tel, 17, 409

Lamers, H. J. G. L. M., \& Leitherer, C. 1993, ApJ, 412, 771

Manchester, R. N., Hobbs, G. B., Teoh, A., \& Hobbs, M. 2005, AJ, 129, 1993

McClure-Griffiths, N. M., Dickey, J. M., Gaensler, B. M., \& Green, A. J. 2001, ApJ, 562, 424

McCray, R., \& Kafatos, M. 1987, ApJ, 317, 190

Noutsos, A., Johnston, S., Kramer, M., \& Karastergiou, A. 2008, MNRAS, 386, 1881

Orellana, R. B., de Biasi, M. S., Bustos Fierro, I. H., \& Calderón, J. H. 2010, A\&A, 521, A39

Perna, R., \& Raymond, J. 2000, ApJ, 539, 706

Perryman, M. A. C., \& ESA 1997, The HiPPARcos and TYCHO catalogues, Astrometric and photometric star catalogues derived from the ESA HipPARcos Space Astrometry Mission, ESA SP, 1200

Röser, S., Schilbach, E., Schwan, H., et al. 2008, A\&A, 488, 401

Sancisi, R. 1974, in Galactic Radio Astronomy, eds. F. J. Kerr, \& S. C. Simonson, IAU Symp., 60, 115

Schaller, G., Schaerer, D., Meynet, G., \& Maeder, A. 1992, A\&AS, 96, 269

Schmidt-Kaler. Th. 1982, in Landolt-Bornstein New Series, Group VI, Vol. 2b, eds. K. Schaifers, \& H. H. Voigt (Berlin: Springer-Verlag)

Tenorio-Tagle, G. 1981, A\&A, 94, 338

Urban, S. E., Wycoff, G. L., \& Makarov, V. V. 2000, AJ, 120, 501

van der Sluys, M. V., \& Lamers, H. J. G. L. M. 2003, A\&A, 398, 181

Vondrak, J. 2004, Serbian Astron. J., 168, 1

Walborn, N. R. 1972, AJ, 77, 312

Walborn, N., \& Fitzpatrick, E. 1990, PASP, 102, 379

Weaver, R., McCray, R., Castor, J., Shapiro, P., \& Moore, R. 1977, ApJ, 218, 377 